\title{
Megastability: Coexistence of a countable infinity of nested attractors in a periodically-forced oscillator with spatially-periodic damping
}

\author{
Julien C. Sprott ${ }^{1}$, Sajad Jafari ${ }^{2}$, Abdul Jalil M. Khalaf ${ }^{3}$, and Tomasz Kapitaniak ${ }^{4, a}$ \\ 1 Department of Physics, University of Wisconsin-Madison, Madison, WI 53706, USA \\ 2 Biomedical Engineering Department, Amirkabir University of Technology, Tehran \\ 15875-4413, Iran \\ 3 Department of Mathematics, Faculty of Computer Science and Mathematics, University \\ Of Kufa, Najaf, Iraq \\ 4 Division of Dynamics, Lodz University of Technology, Stefanowskiego 1/15, 90-924 Lodz, \\ Poland
}

Received 2 February 2017 / Received in final form 7 March 2017

Published online 21 June 2017

\begin{abstract}
In this paper, we describe a periodically-forced oscillator with spatially-periodic damping. This system has an infinite number of coexisting nested attractors, including limit cycles, attracting tori, and strange attractors. We are aware of no similar example in the literature.
\end{abstract}

\section{Introduction}

Recently there has been an increasing effort in constructing new chaotic attractors with pre-designed types of equilibria [1-12]. These systems include dynamical systems with no equilibrium points [13-21], with only stable equilibria [22-27], with curves of equilibria [28-30], with surfaces of equilibria [8,9], and with non-hyperbolic equilibria $[31,32]$. Many of these examples belong to a new category of dynamical systems with hidden attractors [33-42]. That may be the reason such systems were rarely found in the past, and only recently have such examples been reported in the literature. However, all the mentioned efforts have focused on the structure and characteristics of their equilibria, while there are other important features in chaotic systems such as the shape and topology of the strange attractor [43-45]. Designing chaotic systems with desired properties unrelated to equilibria has progressed recently with the help of fast computers. Some examples are chaotic systems with different kinds of symmetry [46-48], with multi-scroll attractors [17,49-56], and with the algebraically simplest equations [57-61].

One important category of chaotic systems includes periodically-forced nonlinear oscillators [62], of which the van der Pol system in one of the oldest [63-66]. This system is actually a linear oscillator but with a spatially-dependent nonlinear damping.

\footnotetext{
${ }^{a}$ e-mail: tomasz.kapitaniak@p.lodz.pl
} 


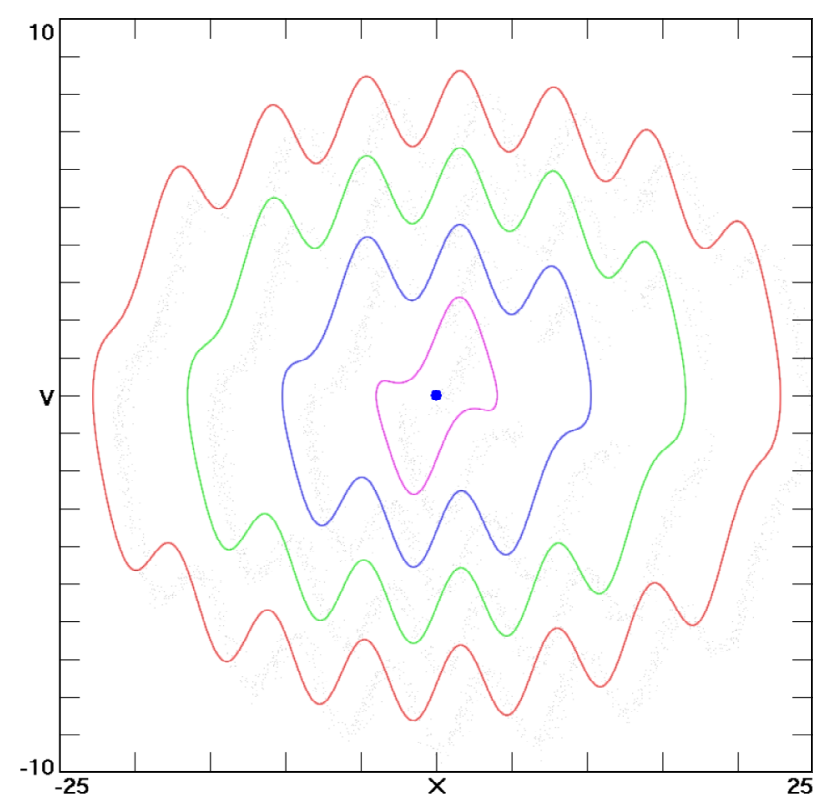

Fig. 1. The first four limit cycles of System (1) for initial conditions $\left(x_{0}, v_{0}\right)=(n \pi, 0)$ with $n=1,3,5,7$.

Multistability is one of the most important phenomena in dynamical systems [67-76] since it occurs in many areas of science including physics, chemistry, biology, economics, and nature. The attracting state of a multistable system depends on the initial conditions in addition to the usual sensitive dependence on initial conditions that characterizes a chaotic system and precludes long-term predictability. Multistability can be undesirable, for example, in the design of a commercial device with specific characteristics where it is necessary to stabilize the desired state in the presence of noise [72]. On the other hand, multistability allows flexibility in the system performance without changing parameters, and that can be used with the right control strategies to induce a switching between different coexisting states [72].

In this paper, we describe a chaotic system similar to the forced van der Pol oscillator but with a spatially-periodic damping. It has an infinite number of nested coexisting hidden attractors of different types including limit cycles, tori, and strange attractors. These coexisting attractors form a layered structure similar to cabbage. We use the term megastability for such a system with an infinite number of attractors.

\section{The proposed system}

Consider the following (2-D) system which is discussed in [77],

$$
\begin{aligned}
\dot{x} & =v \\
\dot{v} & =-x+v \cos x .
\end{aligned}
$$

For small values of $x$, System (1) reduces to the van der Pol equation since $\cos x$ can be Taylor expanded as $\cos x=1-\frac{x^{2}}{2}+\ldots$ However, larger values of $x$ give regions of damping and antidumping that are periodic in $x$ with period $2 \pi$, creating an infinite set of nested limit cycles, the first four of which are shown in Figure 1. These 


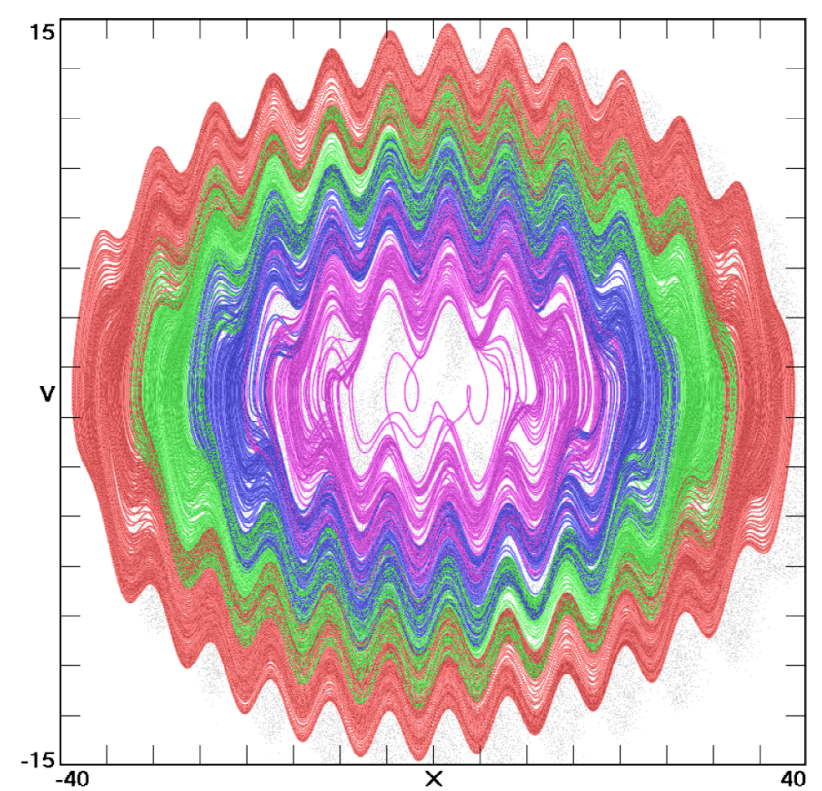

Fig. 2. First four coexisting attractors of System (3) for initial conditions $\left(x_{0}, v_{0}\right)=(n \pi, 0)$ with $n=5,7,9,11$.

attractors are accessed by choosing initial values of $x_{0}$ that are odd multiples of $\pi$ with an initial velocity of $v_{0}=0$ with the result that the orbit is trapped in regions of antidamping bounded by regions of damping. The innermost of these limit cycles resembles the solution of the familiar van der Pol equation.

System (1) has a single equilibrium at the origin $(0,0)$ with eigenvalues of $\frac{1 \pm i \sqrt{3}}{2}$ which means it is an unstable focus. Initial conditions in its vicinity spiral outward to the first limit cycle, making that attractor self-excited. However, all the other limit cycles are hidden since their basins of attraction do not include the neighborhood of any equilibria.

As with the van der Pol equation, a temporally-periodic forcing function can be added to System (1), giving a system whose most general form is

$$
\begin{aligned}
& \dot{x}=v \\
& \dot{v}=-\omega^{2} x+v \cos x+A \sin (\Omega t) .
\end{aligned}
$$

It was earlier noted that this system can have chaotic solutions for an appropriate choice of parameters [62]. Here we take $\omega=0.33, A=1$, and $\Omega=0.73$, which is one of many islands of chaos in the 3 -D parameter space with dynamics typical of the other such regions but with an especially large Kaplan-Yorke dimension. The resulting system is given by

$$
\begin{aligned}
& \dot{x}=v \\
& \dot{v}=-(0.33)^{2} x+v \cos x+\sin (0.73 t) .
\end{aligned}
$$

Figure 2 shows the coexisting attractors for System (3). The innermost attractor is chaotic with Lyapunov exponents of $(0.0063,0,-0.0573)$ and a Kaplan-Yorke dimension of 2.1100. The next one is weakly chaotic with Lyapunov exponents of $(0.0005,0,-0.0162)$ and a Kaplan-Yorke dimension of 2.0317, and the rest are 


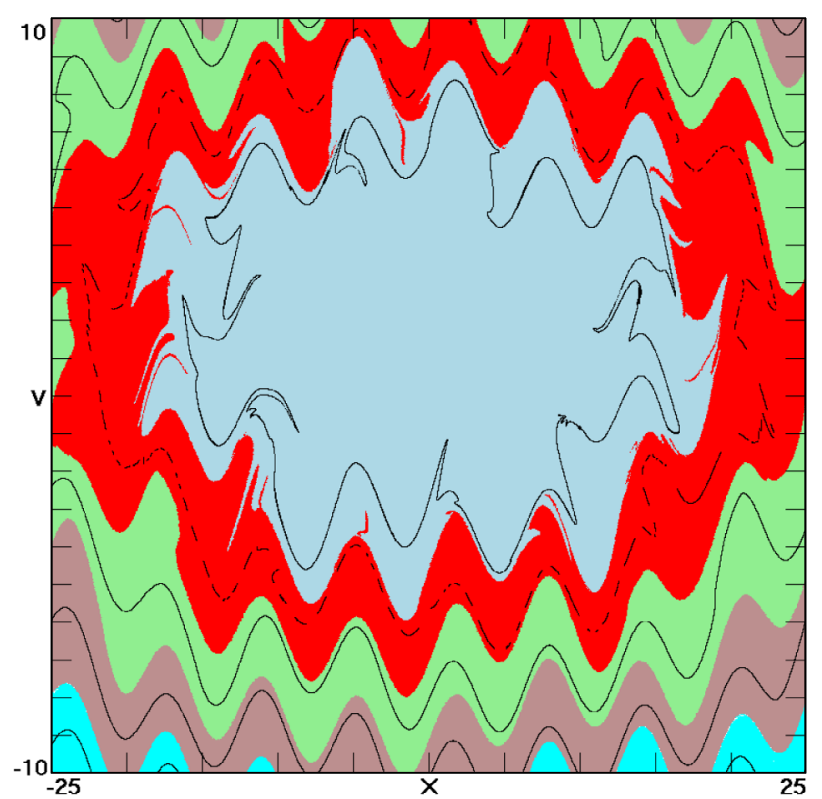

Fig. 3. Poincaré section of the first five coexisting attractors of System (3) in the plane $(t \bmod 2 \pi)=\pi$ with their respective basins of attraction in color.

attracting tori with two zero Lyapunov exponents and one negative one. The negative exponent is given by the time average of $\cos x$ along the orbit and approaches zero as the orbit approaches a circle for large values of $x$. The layered structure resembles a red cabbage.

Another way to visualize the nested attractors is through a plot of their basins of attraction as shown in Figure 3. This plot shows a Poincaré section the first five attractors at values of time given by $(t \bmod 2 \pi)=\pi$ with cross sections of their respective basins in color. The basin boundaries for the tori are relatively smooth, but the basin boundary for the two inner strange attractors appear to have some fractal structure.

Yet another way to visualize the multiple attractors is through a plot of the Lyapunov exponents (LEs), Kaplan-Yorke dimension $\left(D_{K Y}\right)$, and maximum values of $x\left(x_{m}\right)$ as a function of the initial value of $x\left(x_{0}\right)$ for $v_{0}=0$ as shown in Figure 4a. Five attractors of successive larger size are clearly indicated with an abrupt transition between them as the basin boundaries are crossed. The positive largest LE and $D_{K Y}>2$ confirm that the first two are chaotic, while the others are quasiperiodic as indicated by the two zero Lyapunov exponents and the Kaplan-Yorke dimension of 2.0.

\section{Bifurcation analysis}

It is instructive to examine the transition from System (1) to System (3) by using the forcing amplitude $A$ in equation (2) as a bifurcation parameter with initial conditions $\left(x_{0}, v_{0}\right)=(\pi, 0)$. Figure $4 \mathrm{~b}$ shows such a plot. For $A>0$ but small, the limit cycle nearest the origin becomes a thin torus that grows in size until the two frequencies suddenly lock around $A=0.209$ forming a limit cycle. The limit cycle increases in size and period until it collides with the surrounding torus and becomes chaotic around $A=0.65$. The resulting strange attractor continues to increase in size, but with quasiperiodic windows until it merges with the next larger torus around 

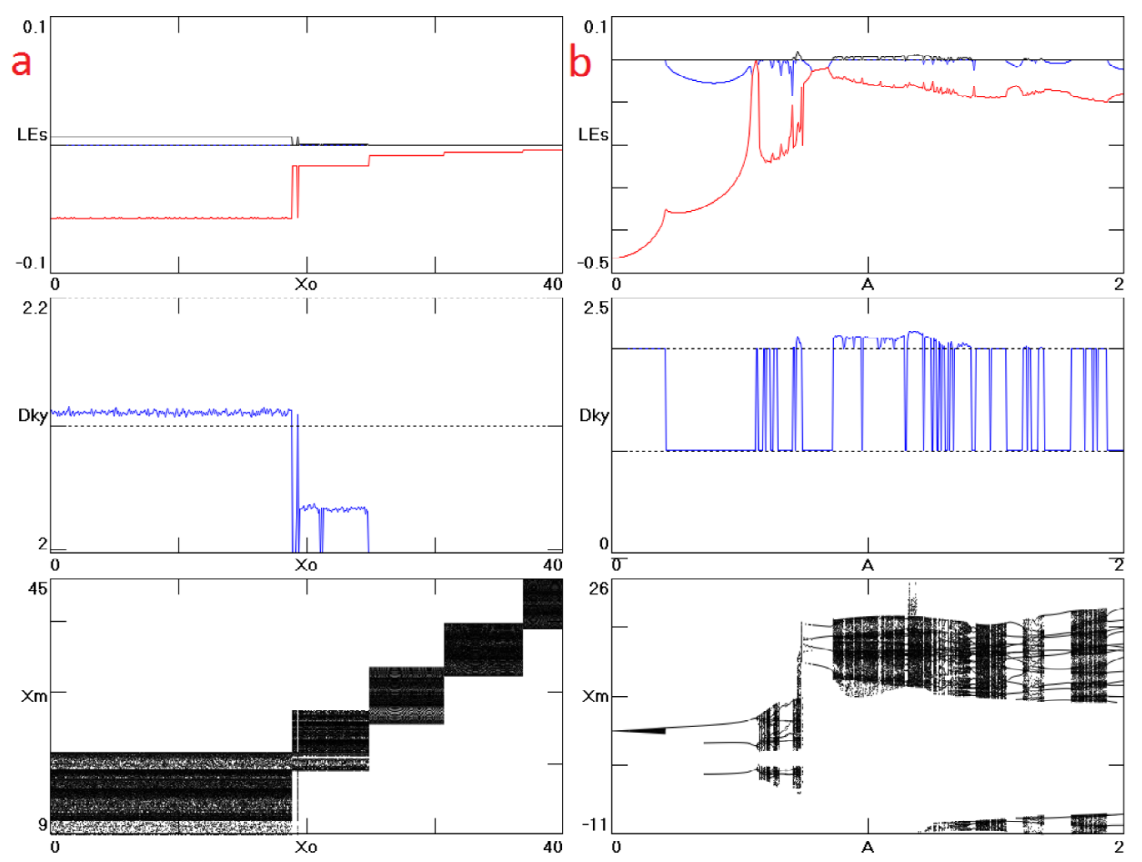

Fig. 4. (a) Lyapunov exponents, Kaplan-Yorke dimension, and maximum values of $x$ versus initial value of $x$ for System (3) with $v_{0}=0$. (b) Lyapunov exponents, Kaplan-Yorke dimension, and maximum values of $x$ versus forcing amplitude $A$ for the innermost attractor of System (2) with $\omega=0.33$ and $\Omega=0.73$.

$A=0.723$ giving birth to a larger strange attractor that persists to $A=1$ and beyond with quasiperiodic windows.

\section{Conclusion}

Unlike the recent effort in constructing new chaotic attractors with pre-designed types of equilibria, designing chaotic systems with other desired properties has attracted less attention. Fortunately, there is still some limited research on designing chaotic systems with other features unrelated to their equilibria. In this paper, we described a megastable, chaotic, periodically-forced, nonlinear oscillator and showed that it can have an infinite number of coexisting attractors, including limit cycles, attracting tori, and strange attractors with a topology not previously reported. Most of these attractors are hidden. A more detailed analysis including exploration of the $(\omega, \Omega)$ plane would be a worthy extension of the work.

One of us (TK) has been supported by the Polish National Science Centre, MAESTRO Programme - Project No 2013/08/A/ST8/00/780.

\section{References}

1. Z. Wei, Phys. Lett. A 376, 102 (2011)

2. X. Wang, G. Chen, Commun. Nonlinear Sci. Numer. Simul 17, 1264 (2012)

3. S. Jafari, J. Sprott, Chaos Solitons Fractals 57, 79 (2013) 
4. X. Wang, G. Chen, Nonlinear Dyn. 71, 429 (2013)

5. Y. Chen, Q. Yang, Math. Comput. Simul. 112, 40 (2015)

6. T. Gotthans, J. Petrela, Nonlinear Dyn. 81, 1143 (2015)

7. J.C. Sprott, S. Jafari, V.-T. Pham, Z.S. Hosseini, Phys. Lett. A 379, 2030 (2015)

8. S. Jafari, J. Sprott, M. Molaie, Int. J. Bifurc. Chaos 26, 1650098 (2016)

9. S. Jafari, J.C. Sprott, V.-T. Pham, C. Volos, C. Li, Nonlinear Dyn. 86, 1349 (2016)

10. V.-T. Pham, S. Jafari, C. Volos, A. Giakoumis, S. Vaidyanathan, T. Kapitaniak, IEEE Transactions on Circuits and Systems II: Express Briefs, in Press (2016)

11. V.-T. Pham, S. Jafari, C. Volos, S. Vaidyanathan, T. Kapitaniak, Opt. Int. J. Light Electron Opt. 127, 9111 (2016)

12. V.-T. Pham, S. Jafari, X. Wang, J. Ma, Int. J. Bifurc. Chaos 26, 1650069 (2016)

13. S. Jafari, J.C. Sprott, S.M.R. Hashemi Golpayegani, Phys. Lett. A 377, 699 (2013)

14. S. Jafari, J.C. Sprott, V.-T. Pham, S.M.R. Hashemi Golpayegani, A.H. Jafari, Int. J. Bifurc. Chaos 24, 1450134 (2014)

15. V.-T. Pham, C. Volos, S. Jafari, Z. Wei, X. Wang, Int. J. Bifurc. Chaos 24, 1450073 (2014)

16. F.R. Tahir, S. Jafari, V.-T. Pham, C. Volos, X. Wang, Int. J. Bifurc. Chaos 25, 1550056 (2015)

17. S. Jafari, V.-T. Pham, T. Kapitaniak, Int. J. Bifurc. Chaos 26, 1650031 (2016)

18. V.-T. Pham, S. Vaidyanathan, C. Volos, S. Jafari, S.T. Kingni, Opt. Int. J. Light Electron Opt. 127, 3259 (2016)

19. V.-T. Pham, S. Vaidyanathan, C. Volos, S. Jafari, N.V. Kuznetsov, T.-M. Hoang, Eur. Phys. J. Special Topics 225, 127 (2016)

20. Z. Wei, Y. Tang, H. Chen, I. Pehlivan, Optoelectron. Adv. Mater. Rapid Commun 7, $984(2013)$

21. Z. Wei, R. Wang, A. Liu, Math. Comput. Simul 100, 13 (2014)

22. M. Molaie, S. Jafari, J.C. Sprott, S.M.R. Hashemi Golpayegani, Int. J. Bifurc. Chaos 23, 1350188 (2013)

23. S.T. Kingni, S. Jafari, H. Simo, P. Woafo, Eur. Phys. J. Plus 129, 1 (2014)

24. S.-K. Lao, Y. Shekofteh, S. Jafari, J.C. Sprott, Int. J. Bifurc. Chaos 24, 1450010 (2014)

25. V.-T. Pham, C. Volos, S. Jafari, X. Wang, Optoelectron. Adv. Mater. Rapid Commun. 8, $535(2014)$

26. Z. Wei, I. Moroz, A. Liu, Turk. J. Math 38, 672 (2014)

27. Z. Wei, W. Zhang, Int. J. Bifurc. Chaos 24, 1450127 (2014)

28. S. Jafari, J.C. Sprott, Chaos Solitons Fractals 57, 79 (2013)

29. S.T. Kingni, V.-T. Pham, S. Jafari, G.R. Kol, P. Woafo, Circuits, Syst. Signal Process. 35, 1933 (2016)

30. V.T. Pham, S. Jafari, C. Volos, S. Vaidyanathan, T. Kapitaniak, Opt. Int. J. Light Electron Opt. (2016)

31. Z. Wei, J. Sprott, H. Chen, Phys. Lett. A 379, 2184 (2015)

32. Z. Wei, W. Zhang, M. Yao, Nonlinear Dyn. 82, 1251 (2015)

33. N. Kuznetsov, G. Leonov, V. Vagaitsev, IFAC Proceedings Volumes (IFACPapersOnline) 4, 29 (2010)

34. G. Leonov, N. Kuznetsov, From Physics to Control Through an Emergent View (World Scientific, 2010), Vol. 15

35. V. Bragin, V. Vagaitsev, N. Kuznetsov, G. Leonov, J. Comput. Syst. Sci. Int. 50, 511 (2011)

36. N. Kuznetsov, G. Leonov, S. Seledzhi, IFAC Proceedings Volumes (IFAC-PapersOnline) 18, 2506 (2011)

37. G. Leonov, N. Kuznetsov, in Doklady Mathematics (Springer, 2011), p. 475

38. G. Leonov, N. Kuznetsov, IFAC Proceedings Volumes (IFAC-PapersOnline) 18, 2494 (2011)

39. G. Leonov, N. Kuznetsov, O. Kuznetsova, S. Seledzhi, V. Vagaitsev, Trans. Syst. Control 6, 54 (2011)

40. G. Leonov, N. Kuznetsov, V. Vagaitsev, Phys. Lett. A 375, 2230 (2011) 
41. G. Leonov, N. Kuznetsov, T. Mokaev, Eur. Phys. J. Special Topics 224, 1421 (2015)

42. G. Leonov, N. Kuznetsov, T. Mokaev, Commun. Nonlinear Sci. Numer. Simul. 28, 166 (2015)

43. R. Gilmore, M. Lefranc, The Topology of Chaos: Alice in Stretch and Squeezeland (John Wiley \& Sons, 2012)

44. R. Gilmore, Rev. Mod. Phys. 70, 1455 (1998)

45. T.D. Tsankov, R. Gilmore, Phys. Rev. E 69, 056206 (2004)

46. C. Li, W. Hu, J.C. Sprott, X. Wang, Eur. Phys. J. Special Topics 224, 1493 (2015)

47. J.C. Sprott, Int. J. Bifurc. Chaos 25, 1550078 (2015)

48. J.C. Sprott, Int. J. Bifurc. Chaos 24, 1450009 (2014)

49. W.K. Tang, G. Zhong, G. Chen, K. Man, IEEE Trans. Circuits Syst. 48, 1369 (2001)

50. J. Lu, G. Chen, Int. J. Bifurc. Chaos 16, 775 (2006)

51. L. Wang, Nonlinear Dyn. 56, 453 (2009)

52. J. Ma, X. Wu, R. Chu, L. Zhang, Nonlinear Dyn. 76, 1951 (2014)

53. J. Munoz-Pacheco, E. Tlelo-Cuautle, I. Toxqui-Toxqui, C. Sanchez-Lopez, R. TrejoGuerra, Int. J. Electron. 101, 1559 (2014)

54. T. Zuo, K. Sun, X. Ai, H. Wang, IEEE Transactions on Circuits and Systems II: Express Briefs 61, 818 (2014)

55. E. Tlelo-Cuautle, J. Rangel-Magdaleno, A. Pano-Azucena, P. Obeso-Rodelo, J. Nunez-Perez, Commun. Nonlinear Sci. Numer. Simul. 27, 66 (2015)

56. F. Li, C. Yao, Nonlinear Dyn. 84, 2305 (2016)

57. C.D. Brummitt, J. Sprott, Phys. Lett. A 373, 2717 (2009)

58. H. Gottlieb, J. Sprott, Phys. Lett. A 291, 385 (2001)

59. J.C. Sprott, S.J. Linz, Int. J. Chaos Theory Appl. 5, 1 (2000)

60. J.C. Sprott, Am. J. Phys. 68, 758 (2008)

61. J.C. Sprott, Phys. Rev. E 50, R647 (1994)

62. J.C. Sprott, Elegant Chaos: Algebraically Simple Chaotic Flows (World Scientific, 2010)

63. B. Van der Pol, Radio Rev. 1, 701 (1920)

64. B. Van der Pol, The London, Edinburgh, and Dublin Philosophical Magazine and Journal of Science 2, 978 (1926)

65. M.L. Cartwright, J.E. Littlewood, J. London Math. Soc. 1, 180 (1945)

66. N. Levinson, Ann. Math. 50, 127 (1949)

67. S. Yanchuk, T. Kapitaniak, Phys. Lett. A 290139 (2001)

68. S. Yanchuk, T. Kapitaniak, Phys. Rev. E 64, 056235 (2001)

69. T.J. Kapitaniak, Sound Vib. 102, 440 (1985)

70. Y. Maistrenko, T. Kapitaniak, P. Szuminski, Phys. Rev. E 56, 6393 (1997)

71. A. Silchenko, T. Kapitaniak, V. Anishchenko, Phys. Rev. E 591593 (1999)

72. A.N. Pisarchik, U. Feudel, Phys. Rep. 540, 167 (2014)

73. A. Chudzik, P. Perlikowski, A. Stefanski, T. Kapitaniak, Int. J. Bifurc. Chaos 21, 1907 (2011)

74. P. Jaros, P. Perlikowski, T. Kapitaniak, Eur. Phys. J. Special Topics 224, 1541 (2015)

75. T. Kapitaniak, G.A. Leonov, Eur. Phys. J. Special Topics 224, 1405 (2015)

76. P. Jaros, T. Kapitaniak, P. Perlikowski, Eur. Phys. J. Special Topics 225, 2623 (2014)

77. P.B. Kahn, Y. Zarmi, Nonlinear Dynamics: Exploration Through Normal Forms (Courier Corporation, 2014)

Open Access This is an Open Access article distributed under the terms of the Creative Commons Attribution License (http://creativecommons.org/licenses/by/4.0), which permits unrestricted use, distribution, and reproduction in any medium, provided the original work is properly cited. 